\title{
Motivi di ecfrasi dantesca in Michelangelo secondo Benedetto Varchi: ancora sulle Due lezzioni all'Accademia fiorentina
}

\author{
Alessandra Tramontana \\ Università degli Studi di Messina \\ alessandra.tramontana@unime.it
}

\section{Riassunto}

Il saggio si propone di individuare la peculiare prospettiva dell'umanista Benedetto Varchi in relazione all'uso che della Commedia dantesca fa Michelangelo Buonarroti. Nelle due prolusioni dal titolo Due lezzioni, recitate presso l'Accademia fiorentina nel 1547, egli infatti delinea il profilo di un artista, Michelangelo appunto, che si è nutrito delle pagine dantesche, ma ha pure fatto suo il profilo etico e civile del grande poeta medievale. E proprio in virtù di questo costante modello ha realizzato i suoi capolavori (poetici e artistici) trasfigurando secondo tecniche ecfrastiche l'opera dantesca.

Parole chiave: Rinascimento; ecfrasi; Benedetto Varchi; Michelangelo Buonarroti; Dante.

\section{Abstract. Motifs of Dante's ekphrasis in Michelangelo according to Benedetto Varchi: again on the Due lezzioni at the Florentine Academy}

The essay aims to identify the peculiar perspective of the humanistic Benedetto Varchi in relation to the use that Michelangelo Buonarroti makes of Dante's Comedy. In the two lecture notes intitled Due lezzioni, recited at the Florentine Academy in 1547, he in fact outlines the profile of an artist, Michelangelo in fact, who fed on Dante's pages, but he also made the ethical and civil profile of the great medieval poet his own. And precisely by virtue of this constant model he created his masterpieces (poetic and artistic), transfiguring Dante's work according to ekphrastic techniques.

Keywords: Renaissance; ekphrasis; Benedetto Varchi; Michelangelo Buonarroti; Dante. 
Quando nel 1764 Francesco Algarotti nel suo Saggio sopra la pittura scrive che "quello spirito bizzarro e profondo di Michelangelo nelle sue composizioni danteggia, come omerizzavano altre volte Fidia e Apelle" (Algarotti, 1764, pp. 79-80), sintetizza con efficacia il fulcro di un dibattito, che, originato dal celebre ut pictura poesis oraziano, venne declinato secondo rinnovati principi nel corso del Cinquecento, proprio in funzione del polimorfico ingegno del Buonarroti. ${ }^{1}$

Il binomio Dante-Michelangelo, alimentato dalla fama di "gran dantista" 2 che l'artista riscuoteva tra i contemporanei, costituì infatti un motivo topico di tanti scritti del tempo, ${ }^{3}$ sollecitato dall'innegabile fascino che, anche per affinità biografiche e culturali, il grande fiorentino dovette esercitare su un "omo sanza lettere" e tuttavia a lungo dedito alla lettura della poesia dantesca. ${ }^{4}$ Degli esiti che tale costante esercizio sortì in Michelangelo versificatore, "che molto lo [Dante] amirava et imitava ne' concetti e nelle invenzioni" (Vasari, 1962, vol. 1, p. 120), fa fede pure un unanime e pubblico riconoscimento post mortem da parte dei contemporanei in occasione della maestosa cerimonia funebre celebrata a Firenze nella chiesa di San Lorenzo nel luglio 1564. L'Accademia del Disegno, allora di recente istituzione, fece allestire infatti intorno al catafalco statue e dipinti che, accanto alle arti canoniche della pittura, della scultura e dell'architettura nelle quali il Buonarroti aveva primeggiato, effigiassero anche la poesia con un quadro in cui Michelangelo, intento a comporre, appariva circondato dalle Muse e da Apollo nell'atto di incoronare l'artista con l'alloro; il motto era un luogo dantesco: "conducemi Apollo / et nove Muse mi dimostran l'Orse" (Par. II, 8-9). ${ }^{5} \mathrm{E}$ anche laddove di Michelangelo si intendeva esaltare il genio artistico, la presenza dantesca appariva naturale complemento. Lo attesta il soggetto della tela di Alessandro Allori, durante la celebrazione funebre ubicata accanto all'altare maggiore, che raffigura l'artista nei campi Elisi attorniato da ambo i lati rispettivamente da pittori e scultori antichi e moderni, i quali

gli erano intorno, in quel modo stesso che ricevettero Virgilio gli altri poeti nel suo ritorno, secondo la finzione del nostro divin poeta Dante, dal quale

1. Rensselaer Lee, 1940, pp. 211-212, nota come già Leon Battista Alberti un secolo prima sottolineasse l'influsso esercitato da Omero su Fidia nella rappresentazione della maestà del dio greco Zeus.

2. Così lo apostrofa Donato Giannotti nei Dialogi [...], dé giorni che Dante consumò nel cercare l'Inferno e 'l Purgatorio, 1939, p. 40.

3. Per una carrellata di testimonianze cinquecentesche vd. P. Barocchi in Vasari, 1962, vol. 4, pp. 1982-1988.

4. Vd. a proposito quanto documenta la Dubard de Gaillarbois in Varchi, 2020, p. 164, nota 443. Sui limiti di un insistito accostamento tra i due personaggi nei letterati del tempo avverte Fiorato, 1990.

5. A una dettagliata descrizione della cerimonia fu dedicato un libretto pubblicato anonimo a Firenze presso i Giunti nel 1564 (sul quale: Fratini, 2015). A proposito dei quattro quadri accompagnati da altrettante sculture vd. Esequie, 1875, pp. 44-46. Sul significato anche politico di tale allestimento funebre: Corsaro, 2008, pp. 383-384. 
essendosi presa questa invenzione, si tolse anche il verso, che in un breve si leggeva sopra [...]: "Tutti l'ammiran, tutti onor gli fanno" (Inf. IV, 133). ${ }^{6}$

Il senso dell'icastica iconografia veniva decodificato da Benedetto Varchi nella sua Orazione funerale, pronunciata su incarico dell'entourage culturale fiorentino in occasione di tale cerimonia funebre. ${ }^{7}$ Proprio la citata tela di Allori, che era "sopra il capo" dell'oratore, ratificava infatti come Michelangelo con le sue opere "havesse tolto le prime palme di mano" ad antichi e moderni, superandoli tutti. ${ }^{8}$ Pubblicata da Giunti a poca distanza dalla cerimonia, l'orazione di Varchi costituisce, dopo le Due lezzioni, ${ }^{9}$ il secondo atto di un'operazione culturale (ma anche politica) ${ }^{10}$ che lo vide indiscusso protagonista e che, probabilmente proprio perché orbitava attorno a Michelangelo, riscosse molta fortuna. Anche le Due lezzioni infatti, pronunciate rispettivamente il 6 e il 13 marzo 1547 presso l'Accademia fiorentina, pur in assenza del Buonarroti da tempo a Roma, suscitarono entusiasmo e alimentarono dibattiti, incoraggiati pure dalla stampa che due anni più tardi ne avrebbe curato il Torrentino, tipografo ufficiale della corte del Duca di Firenze Cosimo I. ${ }^{11}$

Proprio nell'ultima sezione della seconda lezione varchiana dall'eloquente titolo In che siano simili et in che differenti $i$ poeti et $i$ pittori [...], Varchi riaffrontava la questione oraziana dell'ut pictura poesis e ad esordio dichiarava che "essendo il fine della poesia et della pittura il medesimo secondo alcuni, ciò è imitare la natura quanto possono il più, vengono ad essere una medesima et nobili ad un modo". ${ }^{12}$ E sulla scorta di canoniche auctoritates, a comprova del suo assunto chiamava in causa poco dopo Omero, il poeta per eccellenza:

6. Esequie, 1875, p. 49. Il verso dantesco fa riferimento, com'è noto, ad Aristotele, "'l maestro di color che sanno" (Inf. IV, 131), cui vanno incontro gli spiriti sapienti del Limbo.

7. Non era un incarico inaspettato quello cui Varchi adempì in questa occasione; dal suo rientro a Firenze aveva già recitato ben cinque orazioni funebri, tra cui quella a Santa Maria Novella in onore di Pietro Bembo (febbraio 1547): Varchi, 1546.

8. Orazione funerale, 2008, p. 19 (si è operato qua e là qualche intervento grafico e di interpunzione).

9. Due lezzioni di messer Benedetto Varchi [...] (1549 [ma 1550]).

10. L' "abile regia filomedicea", che a Barocchi, 1981, pp. 17-19, pare messa in atto da Varchi in tale occasione, risulta una forzatura per Lo Re, 2012, il quale sottolinea invece il clima di ostilità diffuso nell'ambiente ducale che accompagnò il progetto 'michelangiolesco' dell'intellettuale fiorentino. E tuttavia proprio tale disagevole atmosfera potrebbe avere innescato il disegno varchiano, finalizzato -- si vedrà -- al raggiungimento di più obiettivi.

11. Scardamaglia, 2017, pp. 115-116, sottolinea come dell'ampia produzione varchiana legata alla sua attività accademica fiorentina solo questi due testi, con protagonista Michelangelo, meritarono una circolazione a stampa in tempi brevi. Sull'interpretazione soprattutto in chiave storico-artistica delle Due lezzioni sono tanti i contributi critici; di seguito qualche indicazione tra i saggi più recenti: Laffranchi, 2000; Sabbatino, 2005; Spagnolo, 2008; Collareta, 2015.

12. Si segue la recente edizione delle Due lezzioni curata dalla Dubard de Gaillarbois (Varchi, 2020), operando tuttavia, oltre a qualche intervento testuale sulla scorta della cinquecentina, pure un generale ammodernamento sul testo proposto dalla studiosa, che fotografa in toto sotto il profilo grafico e di interpunzione la facies della stampa antica; il luogo citato è a p. 412. Alle pp. 511-512 si trova un prospetto bibliografico delle edizioni integrali e parziali dell'opuscolo varchiano, tra le quali quella curata dalla Barocchi: Varchi \& Borghini, 1998, 
si racconta che Xeusi, che fu tanto eccellente, faceva le donne grandi et forzose, seguitando in ciò Homero; et Plinio racconta che Apelle dipinse in modo Diana fra un coro di vergini che sacrificavano, ch'egli vinse i versi d'Homero che scrivevano questo medesimo. ${ }^{13}$

Anzi osava andare al di là dell'ormai canonico confronto inter pares tra artista e poeta, arrivando ad affermare che il pittore Apelle era addirittura riuscito a superare Omero, il suo modello letterario. In tal modo ammetteva di fatto un altro verisimile, eccezionale, moderno 'sorpasso' nella storia delle arti, quello di Michelangelo nei confronti di Dante:

come [Michelangelo] ne' versi l'ha seguitato et imitato, così nello scolpire et dipignere ha giostrato et combattuto seco, et forse fatto a lui alcuna volta, come si legge, che fece Apelle ad Homero. ${ }^{14}$

L'equazione tra antichi e moderni posta da Varchi (Apelle e Omero da una parte, Michelangelo e Dante dall'altra) avvia un paradigma comparativo che si sarebbe rivelato caro ai circoli intellettuali del tempo, ma che soprattutto, nella peculiare prospettiva dell'umanista, avrebbe consentito una singolare rimodulazione del confronto.

Una pur rapida radiografia dell'opuscolo varchiano che nel $1549(\mathrm{~m} . \mathrm{f}$.) approda alla stampa aiuterà a comprendere il senso complessivo dell'iniziativa alla quale concorsero in tanti, a partire dal mercante, intellettuale e mecenate fiorentino Bartolomeo Bettini che finanziò l'impresa, e del quale Torrentino, nell'epistola prefatoria che apre l'edizione a lui dedicata, esplicita l'amichevole rapporto con Varchi e Michelangelo. ${ }^{15} \mathrm{Ma}$ dal paratesto della cinquecentina emerge pure il ruolo di Luca Martini, destinatario di un'epistola di Varchi collocata nella stampa da Torrentino a mo' di cerniera tra la prima e la seconda lezione. ${ }^{16}$ All'illustre patrono delle arti e indubbio trait d'union tra Varchi e Michelangelo, il 14 marzo del 1547 (dunque il giorno successivo alla seconda prolusione accademica) l'umanista scriveva infatti che volentieri per suo tramite inviava al Buonarroti le Due lezzioni, le quali, affrontando rispettivamente il commento del sonetto michelangiolesco Non ha l'ottimo artista alcun concetto e la vexata quaestio del rapporto tra arti figurative e tra pittura e poesia, a buon diritto investivano gli interessi dell'artista, sollecitato anzi a prendere posizione in merito al dibattito. ${ }^{17}$

con la quale per la seconda lezione (pp. 7-84) si è operato un costante confronto.

13. Varchi, 2020, p. 420, anche per le fonti classiche cui Varchi attingeva.

14. Varchi, 2020, pp. 212-214. Felice preambolo e lucido manifesto ideologico, l'osservazione varchiana è strategicamente incastonata nella prima lezione, per segnalare l'uso già dantesco (ma non petrarchesco) del termine 'artista', posto ad esordio del sonetto michelangiolesco commentato (sui temi delle Due lezzioni: infra, p. 85-86).

15. Varchi, 2020, p. 178. Sul Bettini e la sua amicizia con i protagonisti delle Due lezzioni da ultimo: Varchi, 2020, pp. 72 e 106-108.

16. Varchi, 2020, pp. 292-294.

17. Luca Martini, tra i fondatori dell'Accademia degli Umidi (poi Accademia fiorentina), condivideva con Varchi la passione per Dante e, come lascia intendere un luogo delle Due lezzioni (Varchi, 2020, pp. 398-400), pare studiasse la cosmografia dantesca: Varchi, 1960, p. 368. 
L'operazione editoriale mette insieme un organico corpus di testi che danno vita a un prodotto originale e dai contorni netti, specchio dell'audace progetto di Varchi. ${ }^{18}$ Lumanista, infatti, suscitando non poche polemiche, ${ }^{19}$ nella prima prolusione sceglie di analizzare il carme di un artista vivente (circostanza già inedita) e per di più, secondo gli schemi rinascimentali, dedito ad arti 'meccaniche'; per conferire maggiore forza al suo programma arricchisce poi le due lezioni con altri versi michelangioleschi, alcuni dei quali tramandati solo da tale cinquecentina (come lo stesso sonetto oggetto della prima lezione), dando forma di fatto alla prima antologia di rime del Buonarroti. ${ }^{20}$ Affronta poi nelle prime due sezioni della seconda lezione il tema del 'paragone' tra le arti e con modalità atipiche investe del problema un gruppo di otto maestri d'arte, il cui parere in forma epistolare correda in calce la stampa, suggellata dalla celebre lettera di Michelangelo in difesa della scultura. ${ }^{21}$ Si dedica infine

Un breve profilo del Martini, anche in funzione dei fatti che qui si vanno esponendo, in Varchi, 2020, pp. 108-109, la cui curatrice, tuttavia, da una lettera ricava impropriamente che Varchi intendesse far arrivare all'artista la sola seconda lezione e non pure la prima sul sonetto, come invece pare naturale interpretare: "Poscia che a me conviene disgiugnere questa presente materia, quasi membro dal suo capo, da quella che io trattai (hieri furono otto giorni) nella sposizione sopra il sonetto di Michelagnolo, et a voi piace di volergliele a ogni modo mandare a Roma [...]": Varchi, 2020, p. 294 (il corsivo è mio). Allo stesso Martini di lì a poco avrebbe scritto Michelangelo perché si facesse portavoce della sua riconoscenza verso Varchi, il cui "Libretto", cioè la lezione sul sonetto, egli aveva poi ricevuto per tramite del Bettini, verisimilmente in una trascrizione dello stesso Martini: Buonarroti, 2020, Ep. 299, pp. 717-718 (l'epistola è datata tra marzo e aprile 1547). Sempre in modo indiretto e in relazione alle Due lezzioni Michelangelo si sarebbe rivolto a Varchi, facendogli giungere pure suoi versi, in almeno altre due occasioni testimoniate da due lettere a G. F. Fattucci, cappellano di Santa Maria del Fiore e amico dell'artista: l'una, in una doppia redazione dell'aprile-giugno 1547, l'altra del febbraio (?) 1550 (Buonarroti, 2020, Epp. 301-302, pp. 719-720 e Ep. 364, p. 766); a proposito: Dubard de Gaillarbois, 2018, pp. 6-7.

18. Di seguito una descrizione della cinquecentina (le date delle epistole sono da intendersi m.f.): pp. 3-4: Epistola di Lorenzo Torrentino a Bartolomeo Bettini (12 gennaio 1549); pp. 5-6: Epistola di Benedetto Varchi a Don Luigi da Toledo (7 marzo 1546); pp. 7-12: Proemio alla prima lezione; pp. 13-54: prima lezione Sopra il sottoscritto sonetto di Michelagnolo Buonarroti (le pp. 53-54 sono dedicate a un sonetto di Varchi in cui Michelangelo è celebrato come artista e come poeta: infra, p. 90); p. 55: Epistola di Benedetto Varchi a Luca Martini (14 marzo 1546); pp. 56-60: Proemio alla seconda lezione; pp. 61-89: prima disputa Della maggioranza et nobiltà dell'arti; pp. 89-111: seconda disputa Qual sia più nobile, o la scultura o la pittura; pp. 111-117: terza disputa In che siano simili et in che differenti i poeti et i pittori; pp. 118-119: due sonetti "fatte [sic] già de M. Benedetto Varchi, quando si scoperse la cappella di S. Lorenzo", dedicati a Lorenzo Lenzi e a Bartolomeo Bettini; pp. 120-155: Epistole di otto scultori e pittori (G. Vasari, A. Bronzino, I. da Pontormo, B. del Tasso, F. da Sangallo, N. Tribolo, B. Cellini e Michelangelo).

19. Vi si allude a più riprese nel corso della sua prolusione: Varchi, 2020, pp. 194, 196 e 206.

20. Un elenco dei carmi integralmente o parzialmente riportati nella stampa in Buonarroti, 2020, pp. XCVI-XCVII. Sull'originalità dell'operazione varchiana: Varchi, 2020, pp. 59-60.

21. L'epistola, che si legge pure in Buonarroti, 2020, Ep. 303, pp. 720-721, costituisce l'unico scritto meta-artistico del Buonarroti: Dubard de Gaillarbois, 2018, p. 4. Sulla scelta degli artisti operata da Varchi, i quali, dapprima orbitanti intorno all'Accademia fiorentina, dopo la riforma del 1547 furono di fatto esclusi dall'ambiente cosmiano (con l'unica eccezione del Buonarroti): Geremicca, 2017, p. 21. 
nell'ultima disputa, forse troppo a lungo passata in sordina, ad indagare si diceva - il rapporto tra pittura e poesia, un terreno fertile per raccogliere le fila del programma congegnato e rendere finalmente esplicita la sua personale interpretazione della lettura che di Dante ha fatto Michelangelo. ${ }^{22}$

E innegabile che le pagine delle Due lezzioni siano imbevute del mito michelangiolesco fin dal titolo in cui, come è stato notato, il nome dell'artista ricorre ben due volte: ${ }^{23}$ Varchi disegna il profilo di un genio a tutto tondo, operante su più fronti con esiti sempre eccelsi. ${ }^{24}$ Attraverso un'abile argomentazione filosofica ne sostiene la tesi della superiorità della scultura sulla pittura; ne porta ad esempio in modo capillare i capolavori artistici, ma pure gli esiti poetici (con una strategia che proseguirà nell' Orazione funerale); dimostra insomma come lui solo sia riuscito a superare la natura: "tutti gli altri fecero tutto quello, che era, e pareva possibile, che si facesse: Michelagnolo solo tutto quello, che se era, non pareva possibile, che fusse fatto" (Orazione funerale, 2008, p. 39).

Che nelle intenzioni di Varchi l'occasione potesse rivelarsi propizia per una riabilitazione del Buonarroti agli occhi del duca mediceo, emerge con evidenza dal proemio, in cui l'umanista, rammentando che Michelangelo è "nobilissimo cittadino et accademico nostro" a chi mostra disappunto per la sua scelta, accosta strumentalmente l'eccezionalità dell'artista a quella del principe Cosimo I. ${ }^{25}$

Ma più complesse erano in realtà le ragioni che avevano dettato i modi di questa singolare impresa culturale, tutta giocata nel nome di un indiscusso modello, Dante Alighieri, su cui Varchi e Michelangelo convergono appieno. Gli studi dell'Andreoni hanno ben delineato le tappe del dantismo varchiano. Già negli anni dell'esilio padovano l'umanista avvia le lezioni sull'Etica aristotelica presso l'Accademia degli Infiammati, bembiana e antidantista, proprio esordendo con una citazione del poeta fiorentino. ${ }^{26} \mathrm{La}$ precoce riverenza verso l'illustre modello si traduce, tornato a Firenze, in una lectura Dantis dal forte sapore aristotelico, che sull'esempio lucreziano possa legittimare una poesia (ma pure un linguaggio) filosofici. ${ }^{27} \mathrm{E}$ non a caso nella terza disputa della seconda lezione, rimodulando il principio della Poetica aristotelica per il quale non è la forma in cui si scrive (prosa o versi) a definire un filosofo o

22. Per un resoconto critico sui temi affrontati da Varchi nelle due lezioni e sulle posizioni degli artisti che partecipano alla disputa: Varchi \& Borghini, 1998, pp. VII-XVI.

23. Varchi, 2020, p. 47 ss., ma vd. pure Dubard de Gaillarbois, 2018, pp. 3-4 e Ead., 2019, p. 48, che insiste sul carattere polifonico dell'opera, con Varchi e Michelangelo nel ruolo di co-autori.

24. Esplicito a proposito risulta l'emistichio che chiude l'epigrafe varchiana posta accanto al feretro di Michelangelo: "quattuor unus hic est": Esequie, 1875, p. 29.

25. Varchi, 2020, pp. 194-196; vi si avanza pure l'ipotesi che nel generale clima di 'epurazione' del marzo 1547 (sulla vicenda: Plaisance, 2004, p. 439 ss.) la scelta dei temi delle Due lezzioni potesse avere pure il sapore amaro della vendetta (pp. 69-70).

26. Andreoni, 2005, p. 30.

27. Ead., 2012, p. 104 e Ead., 2017, pp. 7-8. 
un poeta (1447b), Varchi afferma: "Dante [...] in molti luoghi tratta le quistione et di teologia et di filosofia et di tutte l'altre scienze, la qual cosa non è da poeti, ma le tratta oltra il numero, con parole et figure et modi di dire poetici" ${ }^{28}$ Nell' Hercolano, poi, che pur pubblicato postumo (1570) contiene idee maturate già a partire dagli anni ' 40 , in rapporto ai generi letterari viene sancita la superiorità di Dante su Omero, cioè dei moderni sugli antichi; ${ }^{29}$ e anche la scelta di leggere presso l'Accademia fiorentina Petrarca e Dante in contemporanea, pur riconoscendo al primo l'essere "lirico $[. .$.$] più perfetto$ che Dante come heroico", nasce dalla salda consapevolezza che meglio è "essere anzi buono heroico che ottimo lirico" (Andreoni, 2012, p. 213). D’altra parte era proprio la frangia repubblicana degli intellettuali fiorentini ad avere riabilitato proprio intorno agli anni ' 40 del Cinquecento il culto dantesco, su cui finì con il convergere pure la linea politica di Cosimo I, improntata a un interessato recupero delle glorie municipali. ${ }^{30}$

Tale coerenza di linee è percepibile già ad esordio delle Due lezzioni, quando nel proemio al commento del sonetto di Michelangelo l'umanista modula il canonico topos modestiae sulla scarsezza dei propri mezzi espressivi sulla falsariga di Par. XXXIII, 55-75:

il qual sonetto ho preso hoggi a dover interpretare per la grandissima dottrina et incredibile utilità che in esso si racchiude, non secondo che ricercano l'altezza et profondità dei grandissimi concetti di lui, ma in quel modo che potranno la bassezza et debolezza delle mie picciolissime forze et volesse Dio che (ubbidendo la mia lingua all'intelletto) potessi mandar fuori pure una sola particella colla voce di quello che io ne sento dentro nel quore. ${ }^{31}$

Ma nel corso delle prolusioni la presenza dantesca, specchio del versatile artista Michelangelo, è capillare e strategicamente studiata, proprio in virtù della polimorfica facies del poeta trecentesco, esperto in ogni branca del sapere, su cui più volte anche altrove il dotto fiorentino ha argomentato. ${ }^{32}$ Inalterato, del resto, permane nell'Orazione funerale il modello della Commedia sulle cui pagine il giovane Michelangelo, a bottega da Domenico Ghirlandaio, si è formato:

spendeva lodevolmente, e utilissimente quando in leggere i prosatori, ma molto più spesso i poeti toscani; e spezialissimamente la mirabilissima comedia

28. Varchi, 2020, pp. 414-416. Sul rilievo che riveste la terza disputa per comprendere la concezione dell'arte di Varchi: Sgarbi, 2015.

29. Andreoni, 2012, p. 211 ss.

30. Con il Varchi anche G. B. Gelli, P. Giambullari e C. Lenzoni avviano un laboratorio dantesco: Fiorato, 1990, pp. 102-103.

31. Varchi, 2020, p. 194.

32. Ad es. in una lezione sul primo canto del Paradiso (vv. 37-39) Varchi sottolinea che "Dante favella in questo luogo non solamente come poeta ed astrologo, ma ancora come filosofo e teologo" (Opere di Benedetto Varchi, 1859, p. 360) e negli scritti filosofici, a proposito dell'abito dei poeti "quanto alle cose e quanto alle parole", di lui dice "che hora è teologo cristiano, hora metafisico, cioè teologo gentile, hora fisico, hora matematico e bene spesso filosofo politico, o vero civile, et alcuna volta artista" (Andreoni, 2012, p. 344). 
dell'unico poeta, dell'unico astrologo, dell'unico filosofo (e per dire ogni cosa in due parole) dell'unico metafisico gentile, e unico teologo cristiano, Dante Alighieri. ${ }^{33}$

In Varchi cioè al pari di una innovativa lettura dell'Alighieri agisce una personale riflessione sul peculiare riuso che di Dante, proprio in nome di un convergente tracciato spirituale, fa Michelangelo soprattutto nelle arti plastiche; progressivamente esplicitata nel corso delle due lezioni, tale disamina darà corso a una parabola crescente il cui compimento naturale appare la terza disputa della seconda lezione.

Dell'ammirazione di Michelangelo verso Dante, mitizzata già presso i contemporanei, molto si è scritto, e superfluo sarebbe ripercorrerne le tappe. ${ }^{34}$ Basti qui accennare ad alcuni tasselli del percorso culturale dell'artista che hanno segnato la sua speciale relazione col poeta fiorentino. Si pensi alla partecipazione nel 1545 ai dotti conversari sulla Commedia da cui il Giannotti avrebbe tratto i suoi Dialogi; ${ }^{35}$ o al sapore dantesco di tante rime dell'artista, già perspicuo per Ascanio Condivi. ${ }^{36}$ Proprio agli anni 1545-46 risalgono certi carmi michelangioleschi di ispirazione civile, la 'sezione politica' della silloge: ${ }^{37}$ ricchi di stilemi danteschi, in essi l'identificazione con il poeta della Commedia è palese e trova il suo suggello nella terzina finale del sonetto 37 , non a caso posto a chiusura dei Dialogi di Giannotti che con gli amici ne aveva sollecitato la declamazione da parte dell'autore. ${ }^{38} \mathrm{Ma}$ è dal verso conclusivo del sonetto 49, "simil uom né maggior non nacque mai", che più incisivo appare l'inno di Michelangelo a Dante, assieme modello etico, civile ed artistico-letterario.

$\mathrm{Su}$ questo fil rouge che lega i due maestri Varchi costruirà attraverso le Due lezzioni, ma pure l'Orazione funerale, la sua interpretazione ecfrastica dell'opera dell'artista, come certo faranno dopo di lui anche altri, che soprattutto nelle pitture della Sistina e del Giudizio universale individueranno una serie di rimandi alla Commedia, presto divenuti canonici e tuttavia in tempi moderni giustamente ridimensionati. ${ }^{39} \mathrm{Ma}$ prima degli altri e con piglio decisamente singolare l'accademico fiorentino fa di Michelangelo l'oggetto di un suggestivo

33. Orazione funerale, 2008, p. 13.

34. Già Lanzi, 1834, pp. 110-111 e Farinelli, 1918, invitano a non spingersi troppo nella ricerca di esatte corrispondenze tra i due personaggi; per una preliminare rassegna e la bibliografia pregressa: Schiavo, 1975; Gizzi, 1995; Corsaro, 2008; Piva, 2010; Gamberini, 2018.

35. Michel-Ange, 2004, p. CII. Nei Dialogi sono pure contenute liriche michelangiolesche: Varchi, 2020, p. 42 e nota 86.

36. Vita di Michelagnolo Buonarroti, 2009, p. 48. La biografia del Condivi (Vita di Michelagnolo Buonarroti, 1553) è la prima a sé stante (quella di Vasari, del 1550, è parte della complessiva raccolta delle Vite), tra l'altro autorizzata dal Buonarroti che ne aveva preso visione.

37. La definizione è di Masi: Buonarroti, 2020, p. 62, dove i carmi sono siglati, nell'ambito della Silloge, con i nn. 17 (epigramma della Notte), 37 e 49 (sonetti 'danteschi'), 48 (madrigale antimediceo); nelle edizioni precedenti (tra cui Michelangelo, 1992, e Michel-Ange, 2004) hanno i nn. 247, 248, 250 e 249.

38. Fiorato,1990, ma si veda pure il commento ad locum nelle edizioni delle Rime citate.

39. Vd. De Campos, 1932, e in generale sulla ripresa di Dante nel pensiero e nell'opera di Michelangelo: Gizzi, 1995. 
percorso intellettuale, innervandone la produzione di massicci registri danteschi e dunque 'ideologizzandolo', così da delineare in chiave artistica l'originale profilo di un eccezionale, moderno alter ego del poeta medievale.

Già la quartina iniziale del sonetto di Michelangelo offre a Varchi gli elementi per definire il processo dell'atto creativo, inteso come capacità immaginativa cui può seguire la concettualizzazione dell'Idea: ${ }^{40}$

Se uno scultore avesse un marmo [...], in quel marmo sono in potenza et si possono cavare di lui tutte le bellezze che si possono immaginare da qualsivoglia ottimo maestro di dare a qualunche figura [...]. Ora se uno scultore lavorando questo marmo [...], non sapesse condurlo a quella perfezione, la quale egli si era immaginata [...], a chi si deve dare la colpa di questo fatto: al marmo o allo scultore? Al marmo certamente no, perché in lui erano in potenza così le belle fattezze che segli devevano dare, come le non belle che gli sono state date. Dunque il difetto sarà del maestro, il quale non harà saputo sprimere collo scarpello quello che egli s'era immaginato collo ingegno, anzi non ubbidendo le mani alla fantasia, harà fatto tutto il contrario di quello che s'era proposto et penato di dover fare. ${ }^{41}$

E poco dopo chiosa: "quello è solo vero Maestro che può perfettamente mettere in opera colle mani quello ch'egli s'è perfettamente immaginato col cervello" ${ }^{42} \mathrm{E}$ che siffatto passaggio dalla potenza all'atto investa tutte le arti, e dunque pure l'esercizio letterario, è ben chiaro a Varchi, che strategicamente nel proemio, si è visto, auspicava un buon esito dell'impresa che si apprestava a intraprendere "ubbidendo la sua lingua all'intelletto": 43 la mano dell'artista Michelangelo e la lingua dello scrittore Varchi, dunque, opportunamente accordate all'intelletto, danno luogo al processo creativo secondo un iter perfettamente congruente. All'umanista si apriva così la strada per una riflessione che contemplava la mutua corrispondenza tra arti figurative e letteratura, spendibile in chiave ecfrastica. Proprio i nessi "mano/intelletto" e "marmo/ concetto", infatti, consentono a Varchi di introdurre una lettura dell'opera artistica di Michelangelo in chiave dantesca. Nel corso del commento del vocabolo 'artista' (con 'concetto' termine chiave della prima quartina) ne è prova l'ardua equazione Apelle-Omero/Michelangelo-Dante già sottolineata,

40. "Non ha l'ottimo artista alcun concetto, / ch'un marmo solo in se non circoscriva / col suo soverchio et solo a quello arriva / la man, che ubbidisce all'intelletto": Varchi, 2020, p. 198. Sul processo creativo secondo Michelangelo: Michelangelo, 1992, p. 15 ss.

41. Varchi, 2020, pp. 202-204. Sulla stessa linea è Condivi: Vita di Michelagnolo Buonarroti, 2009, p. 50. Per Buonarroti quella dell'esecuzione costituiva la fase meno importante dell'atto creativo e una reiterazione di tentativi doveva indurre a desistere, dando luogo al "non-finito", abile escamotage per lasciare aperte le potenzialità (cioè i multiformi esiti) dell'Idea: vd. Michelangelo, 1992, pp. 31-32 e Schiavone, 2013, pp. 154-159.

42. Varchi, 2020, p. 208. Anche qui, ma a proposito della Pietà Bandini, gli fa eco Condivi: "Hora ha per le mani [Michelangelo] un'opera di marmo, qual egli fa à suo diletto, come quello che pieno di concetti, e forza che ogni giorno ne partorisca qualch'uno": Vita di Michelagnolo Buonarroti, 2009, p. 42.

43. Supra, p. 87. 
cui si accompagna pure il ricorso, obbligato, a luoghi danteschi. ${ }^{44} \mathrm{Ma}$ anche altri luoghi delle Due lezzioni, non riconducibili in modo diretto a Michelangelo, sono spia della peculiare rilettura ecfrastica di Varchi; basti pensare alla sagace traduzione dell'illustrazione dei vantaggi della scultura sulla pittura in un'emblematica rappresentazione scultorea della topografia dell'oltretomba dantesco: "et molto meglio si comprende come noi diremo o l'Inferno o 'l Purgatorio di Dante di rilievo che di pittura". ${ }^{45}$

Smarcandosi dalla posizione politico-civile e antimedicea di Michelangelo, che aveva indotto tanti intellettuali del tempo a formulare un parallelismo tra $\mathrm{i}$ due fiorentini nel crisma dell' exul immeritus, ${ }^{46}$ Varchi esalta il dantismo dell'artista nel nome di una "antica purezza et dantesca gravità", che, precipua non delle sole rime, grazie alla sua "altezza et profondità dei grandissimi concetti", diventa la cifra dell'intera opera michelangiolesca ${ }^{47}$ Nuovo e strategico tassello di tale linea esegetica, rispecchiata nella materiale organizzazione del corpus testuale della cinquecentina, cui Varchi non potè essere estraneo, ${ }^{48}$ costituisce il sonetto del dotto fiorentino, posto a mo' di ponte tra prima e seconda lezione, nel quale il mito michelangiolesco è declinato proprio in funzione del sublime esercizio in una pluralità di arti: "Ben vi potea bastar, chiaro scultore / non sol per opra d'incude et martello / haver, ma co' i colori et col pennello / agguagliato, anzi vinto, il prisco honore. / Ma non contento al gemino valore, / c'ha fatto il secol nostro altero et bello, / l'arme et le paci di quel dolce et fello / cantate, che v'impiaga et molce il core [...]" (Varchi, 2020, p. 292).

$\mathrm{Ma}$ ciò che a Varchi da subito sta a cuore sottolineare è l'uniformità d'approccio che il Buonarroti adotta nelle sue composite creazioni; e per farlo richiama all'attenzione del pubblico delle Due lezzioni l'habitus filosofico dell'artista, efficacemente delineato dal Berni, in relazione alla sua attività poetica, nel famoso Capitolo a Fra Bastian dal Piombo: "ho visto qualche sua composizione: son ignorante, e pur direi d'avélle / lette tutte nel mezzo di Platone; / sì ch'egli è nuovo Apollo e nuovo Apelle: / tacete unquanco, pallide vïole / e liquidi cristalli e fiere snelle: / é dice cose voi dite parole" ${ }^{49}$ Lungi dal ripercorrere l'articolata riflessione critica nata intorno alla felice battuta bernesca, ${ }^{50}$ preme qui porre l'accento sul reimpiego di tali versi nelle Due lezzioni, quando Varchi presenta un sonetto amoroso di Michelangelo: "(come

44. Supra, p. 84. Per i versi della Commedia qui chiamati in causa: Varchi, 2020, pp. 215 e 223.

45. Varchi, 2020, p. 400; è un luogo della seconda disputa della lezione Della maggioranza dell'arti, cui seguiva l'evocazione del progetto del Martini accennato supra, nota 17.

46. Sull'opportunità politica di tale scelta di Varchi: Gamberini, 2018, pp. 206-207.

47. Varchi, 2020, p. 194. Anche nell'Orazione funerale il giudizio sulle rime assume una valenza più ampia in virtù dell'alto profilo di Michelangelo, "grave e concettoso di natura": Orazione funerale, 2008, p. 29.

48. Sulla storia delle Due lezzioni dalla loro composizione alla messa a stampa: Varchi, 2020, pp. 69-72.

49. Buonarroti, 2020, Rime comiche, d'occasione e di corrispondenza, 11, pp. 277-281, vv. 25-31; il corsivo è mio.

50. Tra le pagine più recenti: Fiorato 1990, pp. 95-98; Nelson, 2003, pp. 4-23; Barberi Squarotti, 2004, pp. 396-399; Moroncini, 2012; Buonarroti, 2020, pp. XII-XIV. 
disse quello ingegnosissimo poeta di ciance et da trastullo) egli è nuovo Apollo et nuovo Apelle et non dice parole, ma cose, tratte non solo del mezzo di Platone, ma d'Aristotele". ${ }^{51}$ Di un esercizio morale attento alle cose concrete, e dunque sotto il profilo espressivo incurante del vuoto petrarchismo coevo, Buonarroti del resto non faceva segreto. In un'epistola del 1544 all'amico Luigi del Riccio, forse sollecitato ad esprimersi su un sonetto del Giannotti, dopo averne marcato la conformità con i tempi, precisava in modo icastico: "ma perch'io ò cattivo gusto, non posso far manco stima d'un panno fatto di nuovo, benché romagnuolo, che delle veste usate di seta e d'oro che faren parer bello un uom da sarti" ${ }^{52}$ Ma pure l'intervento nella disputa tra pittura e scultura, costruito con sapiente ironia, altro non è che una programmatica dichiarazione, solo apparentemente bonaria, della superiorità del 'fare' sul 'parlare': "basta che, venendo l'una et l'altra da una medesima intelligenza, ciò è scultura et pittura, si può far fare loro una buona pace insieme et lasciar tante dispute, perchè vi va più tempo che a far le figure" (Varchi, 2020, p. 506).

La formula bernesca sarà sfruttata abilmente da Varchi anche nell'Orazione funerale, ove disegnerà il ritratto di un poeta ed artista dalle forti connotazioni filosofiche:

ma come mostrerremo noi secondo la promissione nostra il Buonarroto essere stato fisico, cio è filosofo naturale, e metafisico, cio è filosofo trasnaturale, e divino? Molti sono coloro nobilissimi e perfettissimi ascoltatori, i quali s'avvisano che chiunque non ha non vo dire studiato gramatica, ma apparato le lingue, ò non si sia conventato publicamente negli studii, non possa essere filosofo né naturale, né divino, come se propriamente la filosofia e la sapienza non nella sostanza delle cose, ma ne' significati consistessero delle parole. ${ }^{53}$

Ma è nella disputa In che siano simili et in che differenti i poeti et $i$ pittori che l'operazione varchiana appare più scoperta: per dimostrare come il continuum di riflessione che connota l'esercizio creativo di Michelangelo, concretizzandosi in un'idea di matrice platonica, possa tradursi indifferentemente in un codice verbale o plastico, egli tira in campo Dante, paradigma ecfrastico per eccellenza:

io per me non dubito punto che Michelagnolo, come ha imitato Dante nella poesia, così non l'habbia imitato nell'opere sue, non solo dando loro quella grandezza et maestà che si vede ne' concetti di Dante, ma ingegnandosi ancora di fare quello, o nel marmo o con i colori, che aveva fatto egli nelle sentenze et colle parole (Varchi, 2020, p. 420).

È ormai chiaro il tracciato ideologico di Varchi: una mappatura della profonda affinità elettiva tra i due grandi fiorentini che investe appieno pure le

51. Varchi, 2020, p. 288; non sfugga il parziale smarcamento dal modello bernesco: Varchi rimette in gioco accanto a Platone pure Aristotele, nella sua ottica prezioso modello per una poesia di 'concetto'.

52. Dunque agli occhi di Michelangelo è sempre preferibile un panno rozzo ('romagnuolo') ma nuovo, piuttosto che una veste di seta e d'oro ma usata: Buonarroti, 2020, Ep. 251, p. 685.

53. Orazione funerale, 2008, p. 29. 
invenzioni figurative di Michelangelo. Il concetto di 'modello', da più voci già declinato, assume ora nelle mani di Varchi contorni più fecondi, volti ad accertare, attraverso una pur rapida pennellata di suoi capolavori artistici, l'impiego che il Buonarroti ha fatto "o nel marmo o con i colori" dei testi danteschi, rigenerando in un linguaggio 'altro' aspetti peculiari della scrittura dell'autore della Commedia, quali la gravità, la concettosità, l'energia, la terribilità.

Le prime tessere di tale griglia comparativa sono estrapolate dal capolavoro michelangiolesco in cui più immediate apparivano le corrispondenze con Dante: alle figure demoniache di Caronte e Minosse dipinte nel Giudizio universale della Sistina Varchi accosta con funzione di archetipo ispiratore le celebri terzine dell' Inferno dedicate ai due mostri, ${ }^{54}$ dando vita a un topos che, di lì a poco riproposto da Vasari e Condivi nella loro Vita michelangiolesca, ${ }^{55}$ avrebbe riscosso tanta fortuna. Ma è con la giovanile Pietà Vaticana, chiamata in causa subito dopo, che l'ut pictura poesis cede il passo ad una più potente ut sculptura poesis:

et chi vede la sua Pietà non vede egli in un marmo viva et vera quella sentenza di quel verso che mostrò Dante non meno pittore che poeta? "Morti gli morti, e' vivi parean vivi" [Purg. XII, 67]. E se alcuno bramasse di vedere come si possano descrivere le figure che dipigne Michelagnolo, non meno poeta che pittore, legga Dante quasi per tutto, ma particolarmente nel canto X et nel XII del Purgatorio (Varchi, 2020, p. 422).

Emerge intanto la ripresa dell'antico motivo della vividezza delle statue di figura umana, con un sotteso confronto dello scultore con Dio, ancor più esplicita nell'Orazione funerale:

sono queste due immagini, l'una viva (benchè afflittissima) e l'altra morta, e hanno tanto in sé l'una del vivo e l'altra del morto, che chiunche le vede pensa ò di vedere essa Verginemaria, ed esso Cristo in carne e in ossa, ò almeno la loro effigie non fatta di marmo da mano mortale, ma discesa divinamente dal Paradiso (Orazione funerale, 2008, p. 20).

Ma il messaggio varchiano si arricchisce in realtà di nuove implicazioni. Il verso dantesco selezionato, infatti, si pone a suggello della serie di exempla di umiltà e superbia punita rappresentati su formelle di marmo bianco che istoriano le pareti della prima cornice, oggetto della prima sezione del XII canto del Purgatorio. Sposando appieno il canone dantesco che conferisce valore a un'opera d'arte se fedele imitazione della realtà naturale, in tale ipotiposi Varchi scorge la maestria 'pittorica' di Dante, che attraverso il paradigma esemplare del "visibile parlare" 56 sembra addirittura dipingere i bassorilievi purgatoriali. Ma con un suggestivo gioco di rifrazione alla raffigurazione varchiana, saldamente congegnata, di un "Dante non meno pittore che poeta" fa subito eco quella di un "Michelagnolo, non meno poeta che pittore": in

54. Inf. III, 109-11 e V, 4-6; Varchi, 2020, p. 422.

55. Vasari, 1962, vol. 1, pp. 74-79 e Vita di Michelagnolo Buonarroti, 2009, p. 41.

56. Purg. X, 95. Sulla 'storia' e la valenza della iunctura: Collareta, 1997. 
una sorta di interazione osmotica che alimenta la grandezza a tutto campo di entrambi, l'artista infonde nuova vita alla lezione di Dante, i cui versi di contro, insegna l'umanista fiorentino, offrono a chi sa coglierla una privilegiata chiave di accesso all'opera del Buonarroti.

Che a conclusione della prolusione Varchi, sempre nel nome dell' "altissimo concetto" dantesco, ${ }^{57}$ innesti la mitografia di Michelangelo su quella della casata medicea non deve stupire, ove si pensi al particolare momento entro cui Varchi doveva disciplinare il suo intervento in Accademia: dopo sei anni di esilio, infatti, pur al servizio di Cosimo già dal 1543, l'intellettuale tra accuse e calunnie stentava ancora a ritagliarsi uno spazio nel difficile ambiente dell'élite culturale fiorentina. ${ }^{58}$ Parve naturale dunque all'umanista siglare in una rinnovata armonia da un lato il nome dei due artisti fiorentini, l'antico e il moderno (ma volontario) exul, dall'altro quello della Cappella Medicea, cioè della Sagrestia Nuova di San Lorenzo dove Michelangelo aveva lasciato incompiute, dopo la partenza definitiva da Firenze, la statua della Madonna Medici e quelle allegoriche delle Ore. ${ }^{59}$ Nella prospettiva di Varchi se già con la Madonna col Bambino Michelangelo "spresse nel marmo miracolosamente quelle due comparazioni miracolose, l'una nel XXIII del Paradiso [...] e l'altra nel XXX [...]"60 (e si noti come la figura etimologica reiteri la connotazione divina dell'uno e dell'altro 'concetto'), con le Ore la dimensione ecfrastica sub specie dantesca raggiunge l'apice. Il Michelangelo varchiano, infatti

volendo [...] significare che per sepolcro di ciascuno di costoro si conveniva non solo un emisperio, ma tutto 'l mondo, ad uno pose la Notte e 'l Giorno et all'altro l'Aurora e'l Crepuscolo, che gli mettessero in mezzo et coprissero, come quegli fanno, la terra (Varchi, 2020, pp. 424-426).

Tale limpida dichiarazione consente all'oratore di raggiungere un duplice obiettivo. Da una parte egli si fa carico di sancire pubblicamente da parte di Michelangelo il riconoscimento del regime dei Medici, attraverso l'apparato statuario di San Lorenzo investiti dall'artista del ruolo di signori del mondo

57. Varchi, 2020, p. 424.

58. I vari travagli degli anni del suo ritorno a Firenze ben emergono dalle pagine di Andreoni, 2012.

59. Nel 1534 Michelangelo infatti si trasferisce a Roma e non farà più rientro in patria: Buonarroti, 2020, pp. XVII-XXVII. In verità la Madonna Medici, tra le prime statue destinate al complesso di San Lorenzo, sarà collocata nella sagrestia dal Tribolo nel 1559. Su committenza di Leone X nel 1519 Michelangelo progetta una cappella sepolcrale dedicata a Lorenzo il Magnifico e al fratello Giuliano, a Lorenzo duca d'Urbino e a Giuliano duca di Nemours. Porterà a termine le statue allegoriche del Giorno e della Notte, dell'Aurora e del Crepuscolo, che il Tribolo nel 1545 collocherà rispettivamente sul sarcofago del duca Giuliano e su quello del duca Lorenzo. Anche in relazione alle complesse fasi architettoniche della Sagrestia Nuova si veda la ricostruzione di Ruschi, 2007 (sull'apparato marmoreo in particolare pp. 45-46). I due sonetti ecfrastici di Varchi, dedicati a Lorenzo Lenzi e a Bartolomeo Bettini, pubblicati nella cinquecentina (supra, p. 85 nota 18) hanno come argomento le statue dell'Aurora e della Notte: Varchi, 2020, pp. 428-430 e 103-104; Lo Re, 2012, pp. 494-495.

60. Varchi, 2020, p. 424; si tratta di Par. XXIII, 121-123 e XXX, 82-84. 
(malgrado egli, a Roma, continuasse a declinare il reiterato invito a tornare a Firenze). Dall'altra la forzata correlazione di tale realizzazione plastica con il magistero dantesco attraverso una terzina del primo canto del Paradiso, utile pure per un'autocitazione, ${ }^{61}$ permette all'umanista di portare a compimento l'insistito processo di 'varchizzazione' del Buonarroti, la cui biografia umana e intellettuale viene così ripercorsa in chiave ecfrastica fino alle battute finali della prolusione proprio nel nome dell'auctoritas dantesca.

\section{Bibliografia}

Algarotti, F. (1764). Saggio sopra la pittura. Livorno: presso Marco Coltellini.

Andreoni, A. (2005). Benedetto Varchi all'Accademia degli Infiammati. Frammenti inediti e appunti sui manoscritti. Studi rinascimentali, 3, 29-44.

Andreoni, A. (2012). La via della dottrina. Le lezioni accademiche di Benedetto Varchi. Pisa: ETS.

Andreoni, A. (2017). Varchi letterato. Un'indagine su Dante, Petrarca e il classicismo. La rivista, 5, 3-11

Barberi Squarotti, G. (2004). Il Berni: la teoria letteraria e pittorica per antifrasi. Letteratura italiana antica, 5, 385-400.

Barocchi, P. (1981). Palazzo Vecchio: committenza e collezionismo medicei e la storiografia artistica contemporanea. Nouvelles de la republique des lettres, 1, 13-39.

Buonarroti, M. (2020). Rime e lettere, a cura di A. Corsaro e G. Masi. Milano: Bompiani.

Collareta, M. (1997)."Visibile parlare". Prospettiva, 86, 102-104.

Collareta, M. (2015). Nouvelles études sur le paragone entre les arts. Perspective, 1, 153-160.

Corsaro, A. (2008). Michelangelo e i letterati. In Officine del nuovo. Sodalizi fra letterati, artisti ed editori nella cultura italiana fra Riforma e Controriforma. Atti del Simposio Internazionale (Utrecht, 8-10 novembre 2007), a cura di H. Hendrix e P. Procaccioli, pp. 383-425. Manziana: Vecchiarelli

De Campos, D. R. (1932). L'ispirazione del Giudizio Universale di Michelangelo. Illustrazione vaticana, 3, 137-141 e 5, 236-240.

Dialogi di Donato Giannotti, dé giorni che Dante consumò nel cercare l'Inferno e 'l Purgatorio. (1939). Ed. critica a cura di D. R. De Campos. Firenze: Sansoni.

Dubard de Gaillarbois, F. (2018). Le "Due lezzioni” varchiane: una soluzione al dilemma michelangiolesco pubblicare o non pubblicare?. In La letteratura italiana e le arti. Atti del XX Congresso dell'ADI-Associazione degli Italianisti (Napoli, 7-10 settembre 2016), a cura di L. Battistini, V. Caputo, M. De Blasi, G. A. Liberti, P. Palomba, V. Panarella, A. Stabile, pp. 1-12. Roma: Adi editore. doi: http://www.italianisti. it/Atti-di-Congresso?pg=cms\&ext=p\&cms_codsec=14\&cms_codcms=1039

Dubard de Gaillarbois, F. (2019). Michelangelo poeta e filosofo: un'invenzione' varchiana?. In La cultura poetica di Benedetto Varchi, a cura di S. M. Vatteroni, pp. 47-59. Berlin: Freie Universität Berlin.

61. Varchi cita infatti Par. I, 43-45 ("Fatto avea di là mane e di qua sera / tal foce, e quasi tutto era là bianco / quello emisperio, e l'altra parte nera”), cui nel maggio 1545 aveva dedicato la quarta lezione relativa a tale canto: Opere di Benedetto Varchi, 1859, pp. 370-371 e Andreoni 2012, p. 154 ss. 
Due lezzioni di messer Benedetto Varchi [...]. (1549 [ma 1550]). Firenze: Lorenzo Torrentino impressor ducale.

Esequie del diuino Michelagnolo Buonarroti. (1875), a cura di G. Piccini-G. Milanesi. Firenze: Tipografia della Gazzetta d'Italia.

Farinelli, A. (1918). Michelangelo e Dante e altri brevi saggi [...]. Torino: Fratelli Bocca. Fiorato, A. (1990). "Fuss'io pur lui": Michelangelo all'ascolto di Dante. Letture classensi, 19, 87-104

Fratini, D. (2015). Le esequie di Michelangelo: aspetti testuali del libretto a stampa. In Intorno a Michelangelo: eredità e iconografia di un mito. Atti del Convegno internazionale di studi (Arezzo, Palazzo della Fraternita dei Laici-Firenze, Accademia delle Arti del Disegno, 5-6 giugno 2014), a cura di A. Baroni, E. Sartoni. Annali aretini, 23, 777-796.

Gamberini, D. (2018). «Antica purezza e dantesca gravità»: forme dell'appropriazione della poesia di Michelangelo nella Firenze di Cosimo I. Italique, 21, 201-233.

Geremicca, A. (2017). 'Damone' per 'Crisero' e gli altri. Benedetto Varchi e gli artisti (prima e dopo l'Accademia Fiorentina). In Intrecci virtuosi. Letterati, artisti e accademie nell'Italia Centrale tra Cinque e Seicento: Roma e Firenze, a cura di C. Chiummo, A. Geremicca, P. Tosini, pp. 11-26, Roma: De Luca Editori d'arte.

Gizzi, C. (1995). Michelangelo e Dante, in Michelangelo e Dante, a cura di C. Gizzi, pp. 221-232. Milano: Electa.

Laffranchi, M. (2000) La "maggioranza delle arti" di Benedetto Varchi. In Lettere e arti nel Rinascimento. Atti del X Convegno internazionale (Chianciano-Pienza, 20-23 luglio 1998), a cura di L. Secchi Tarugi, pp. 647-658. Firenze: Cesati.

Lanzi, L. (1834). Storia pittorica della Italia [...]. Vol. 1. Firenze: Presso Luigi Piazzini.

Lo Re, S. (2012). Varchi e Michelangelo. Annali della Scuola Normale Superiore di Pisa (Quinta serie), 4 (2), 485-516; 613-614.

Michel-Ange (2004). Rime/Poésies Présentation, traduction et notes de A. C. Fiorato. Parigi: Les Belles Lettres.

Michelangelo (1992). Rime e lettere, a cura di P. Mastrocola. Torino: Utet.

Moroncini, A. (2012). Le rime spirituali di Michelangelo e gli affreschi della cappella Paolina: "Cangiar sorte per sol poter divino". Chroniques italiennes, 23 (2), 1-17.

Nelson, J. K. (2003). Michelangelo, "nuovo Apollo e nuovo Apelle”: il canzoniere mai edito e le sculture a confronto. In Michelangelo: poesia e scultura, a cura di J. K. Nelson, pp. 4-23. Milano: Electa.

Opere di Benedetto Varchi ora per la prima volta raccolte [...]. (1859). (Vol. 2) Trieste: Sezione letteraria-artistica del Lloyd Austriaco.

Orazione funerale di Messer Benedetto Varchi fatta, e recitata da lui pubblicamente nell'essequie di Michelagnolo Buonarroti in Firenze nella chiesa di San Lorenzo [...]. (1564). Firenze. Giunti. Herausgegeben und kommentiert von C. Davis (2008), Fontes 23. doi: http://archiv.ub.uni-heidelberg.de/artdok/volltexte/2008/643

Piva, M. (2010). Talenti complementari e capolavori perduti. La leggenda del naufragio della "Divina Commedia" illustrata da Michelangelo dall'Italia alla Francia. Rivista di Letterature moderne e comparate, 63 (2), 117-148.

Plaisance, M. (2004). L'Accademia e il suo principe. Cultura e politica a Firenze al tempo di Cosimo I e di Francesco de’ Medici. Manziana:Vecchiarelli

Rensselaer Lee, W. (1940). Ut Pictura Poesis: The Humanistic Theory of Painting. The Art Bulletin, 22, 197-269.

Ruschi, P. (2007). La Sagrestia Nuova, metamorfosi di uno spazio. In Michelangelo architetto a San Lorenzo. Quattro problemi aperti, Catalogo della mostra (Firenze, 
Casa Buonarroti, 19 giugno-12 novembre 2007), a cura di P. Ruschi, pp. 15-49. Firenze: Mandragora

Sabbatino, P. (2005). Imitazione e illusione. Leonardo da Vinci, Varchi, Marino, Milizia. Studi rinascimentali, 3, 11-27.

Scardamaglia, V. (2017). Benedetto Varchi e Michelangelo 'scultore di versi'. La Rivista, 5, 115-137.

Schiavo, A. (1975). Dante e Michelangelo (influenza dantesca in un sonetto di Michelangelo). In Dante nel pensiero e nell'esegesi dei secoli XIV e XV. Atti del Convegno di Studi realizzato dal Comune di Menfi [...] (27 settembre-2 ottobre 1970), pp. 479-480. Firenze: Olschki.

Schiavone, O. (2013). Michelangelo Buonarroti. Forme del sapere tra letteratura e arte nel Rinascimento. Firenze: Edizioni Polistampa.

Sgarbi, M. (2015). Ludovico Dolce e la nascita della critica d'arte. Un momento della ricezione della Poetica aristotelica nel Rinascimento. Rivista di estetica, (Nuova serie), 59 (2), LV, 163-182.

Spagnolo M. (2008). Ragionare e cicalare d'arte a Firenze nel Cinquecento: tracce di un dibattito tra artisti e letterati. In Officine del nuovo. Sodalizi fra letterati, artisti ed editori nella cultura italiana fra Riforma e Controriforma. Atti del Simposio internazionale (Utrecht, 8-10 novembre 2007), a cura di H. Hendrix e P. Procaccioli, pp. 1-28 (dell'estratto). Manziana: Vecchiarelli.

Varchi, B. (1546). Orazione funebre sopra la morte del reverendissimo cardinal Bembo. Firenze: per il Doni.

Varchi, B. (1960). Lezzione nella quale si disputa della maggioranza delle arti e qual sia più nobile, la scultura o la pittura. In Trattati d'arte del Cinquecento fra manierismo e controriforma, a cura di P. Barocchi (Vol. 1, pp. 1-82; 357-385). Bari: Laterza.

Varchi, B. \& Borghini, V. (1998). Pittura e scultura nel Cinquecento, a cura di P. Barocchi. Livorno: Sillabe.

Varchi, B. (2020). Deux leçons sur l'art. Traduction et édition critique par F. Dubard de Gaillarbois. Parigi: Classiques Garnier.

Vasari, G. (1962). La vita di Michelangelo nelle redazioni del 1550 e del 1568, curata e commentata da P. Barocchi, 5 voll. Milano/Napoli: Ricciardi.

Vita di Michelagnolo Buonarroti raccolta per Ascanio Condivi de la Ripa Transone (1553). Roma: appresso Antonio Blado Stampatore Camerale.

Vita di Michelagnolo Buonarroti raccolta per Ascanio Condivi de la Ripa Transone (1553). Roma: appresso Antonio Blado Stampatore Camerale. Herausgegeben und kommentiert von C. Davis (2009). Fontes 34. doi: http://archiv.ub.uni-heidelberg. de/artdok/volltexte/2009/714/ 